\title{
J-PLUS: Discovery and characterisation of ultracool dwarfs using Virtual Observatory tools
}

\author{
E. Solano ${ }^{1,2}$, E. L. Martín ${ }^{3}$, J. A. Caballero ${ }^{1}$, C. Rodrigo ${ }^{1,2}$, R. E. Angulo ${ }^{4,5,6}$, J. Alcaniz ${ }^{7}$, M. Borges Fernandes ${ }^{7}$,
} A. J. Cenarro ${ }^{8}$, D. Cristóbal-Hornillos ${ }^{8}$, R. A. Dupke, $7,9,11$, E. Alfaro ${ }^{11}$, A. Ederoclite ${ }^{8,12}$, F. Jiménez-Esteban ${ }^{1,2}$, J. A. Hernandez-Jimenez ${ }^{12,13}$, C. Hernández-Monteagudo ${ }^{8}$, R. Lopes de Oliveiraa ${ }^{14,15,7}$, C. López-Sanjuan ${ }^{8}$, A. Marín-Franch ${ }^{8}$, C. Mendes de Oliveira ${ }^{12}$, M. Moles ${ }^{4}$, A. Orsi ${ }^{4}$, L. Schmidtobreick ${ }^{16}$, D. Sobral ${ }^{17}$, L. Sodré ${ }^{12}$, J. Varela ${ }^{8}$, and H. Vázquez Ramió ${ }^{4}$

(Affiliations can be found after the references)

Received 6 February 2019 / Accepted 7 May 2019

\begin{abstract}
Context. Ultracool dwarfs are objects with spectral types equal to or later than M7. Most of them have been discovered using wide-field imaging surveys. The Virtual Observatory has proven to be very useful for efficiently exploiting these astronomical resources.

Aims. We aim to validate a Virtual Observatory methodology designed to discover and characterise ultracool dwarfs in the J-PLUS photometric survey. J-PLUS is a multiband survey carried out with the wide-angle T80Cam optical camera mounted on the $0.83 \mathrm{~m}$ telescope JAST/T80 in the Observatorio Astrofísico de Javalambre. We make use of the Internal Data Release covering $528 \mathrm{deg}^{2}$.

Methods. We complemented J-PLUS photometry with other catalogues in the optical and infrared using VOSA, a Virtual Observatory tool that estimates physical parameters from the spectral energy distribution fitting to collections of theoretical models. Objects identified as ultracool dwarfs were distinguished from background M giants and highly reddened stars using parallaxes and proper motions from Gaia DR2.

Results. We identify 559 ultracool dwarfs, ranging from $i=16.2$ mag to $i=22.4$ mag, of which 187 are candidate ultracool dwarfs not previously reported in the literature. This represents an increase in the number of known ultracool dwarfs of about $50 \%$ in the region of the sky we studied, particularly at the faint end of our sensitivity, which is interesting as reference for future wide and deep surveys such as Euclid. Three candidates are interesting targets for exoplanet surveys because of their proximity (distances less than $40 \mathrm{pc}$ ). We also analysed the kinematics of ultracool dwarfs in our catalogue and found evidence that it is consistent with a Galactic thin-disc population, except for six objects that might be members of the thick disc.

Conclusion. The results we obtained validate the proposed methodology, which will be used in future J-PLUS and J-PAS releases. Considering the region of the sky covered by the Internal Data Release used in this work, we estimate that 3000-3500 new ultracool dwarfs will be discovered at the end of the J-PLUS project.
\end{abstract}

Key words. surveys - virtual observatory tools - stars: low-mass - brown dwarfs

\section{Introduction}

Ultracool dwarfs (UCDs) are defined as objects with spectral types equal to or later than M7 V. They comprise both the lowest mass hydrogen-fusing stars and brown dwarfs (substellar objects not massive enough to sustain steady hydrogen fusion). The M7 V spectral type marks the beginning of a variety of changes with decreasing effective temperature, in particular the appearance of dust clouds, which makes an increasingly important contribution to the atmospheric chemistry (e.g. Jones \& Tsuji 1997; Helling et al. 2008). UCDs represent about $15 \%$ of the stellar population in the solar neighbourhood (e.g. Henry et al. 2006; Gillon et al. 2016).

While the ages of UCDs favour studies of Galactic kinematics, they also play a relevant role in two other types of research lines. On one hand, their low masses and small radii facilitate the discovery of Earth-size planets orbiting them at separations at which surface water could be liquid. Moreover, their proximity to the Sun (UCDs are intrinsically faint objects that cannot be detected at long distances) opens the door to the detection and characterisation of habitable planets in the solar neighbourhood. Proxima Centauri (Anglada-Escudé et al. 2016), TRAPPIST -1 (Gillon et al. 2017), and Barnard's star (Ribas et al. 2018) are excellent examples of this type of research. There are approximately three times as many $\mathbf{M}$ dwarfs as FGK dwarfs in the Milky Way (Kroupa 2001; Chabrier 2003), and small planets appear to surround M dwarfs three to five times more frequently than Sun-like stars (Dressing \& Charbonneau 2015), which means that they could well represent the most common Earth-size planets in our Galaxy.

On the other hand, some of the youngest $(t \leq 100 \mathrm{Myr})$ UCDs in the field may have masses close to or even below the deuterium-burning limit (10-13 $M_{\text {Jup }}$; Chabrier \& Baraffe 2000; Zhang et al. 2019), which makes them exoplanet analogues. Because these objects are nearby, isolated, and not affected by the glare of the host star, they are ideal laboratories for detailed studies of the cool, low-gravity, and dusty atmospheres typical of exoplanets (Faherty et al. 2013; Caballero 2018).

The main sources of UCD discoveries, which now include over $2000 \mathrm{~L}$ and $\mathrm{T}$ dwarfs and several thousand late-M dwarfs (Smart et al. 2017), have been wide-field, optical, and infrared imaging surveys such as the Deep Near Infrared Survey of the Southern Sky (Epchtein et al. 1999), the Sloan Digital Sky Survey (York et al. 2000), the Two Micron All Sky Survey (Skrutskie et al. 2006), the UKIRT Infrared Deep Sky Survey (Lawrence et al. 2007), the Wide-Field Infrared Survey Explorer (Wright et al. 2010), and the Visible and Infrared Survey Telescope for Astronomy (Cross et al. 2012). In the near future, a 


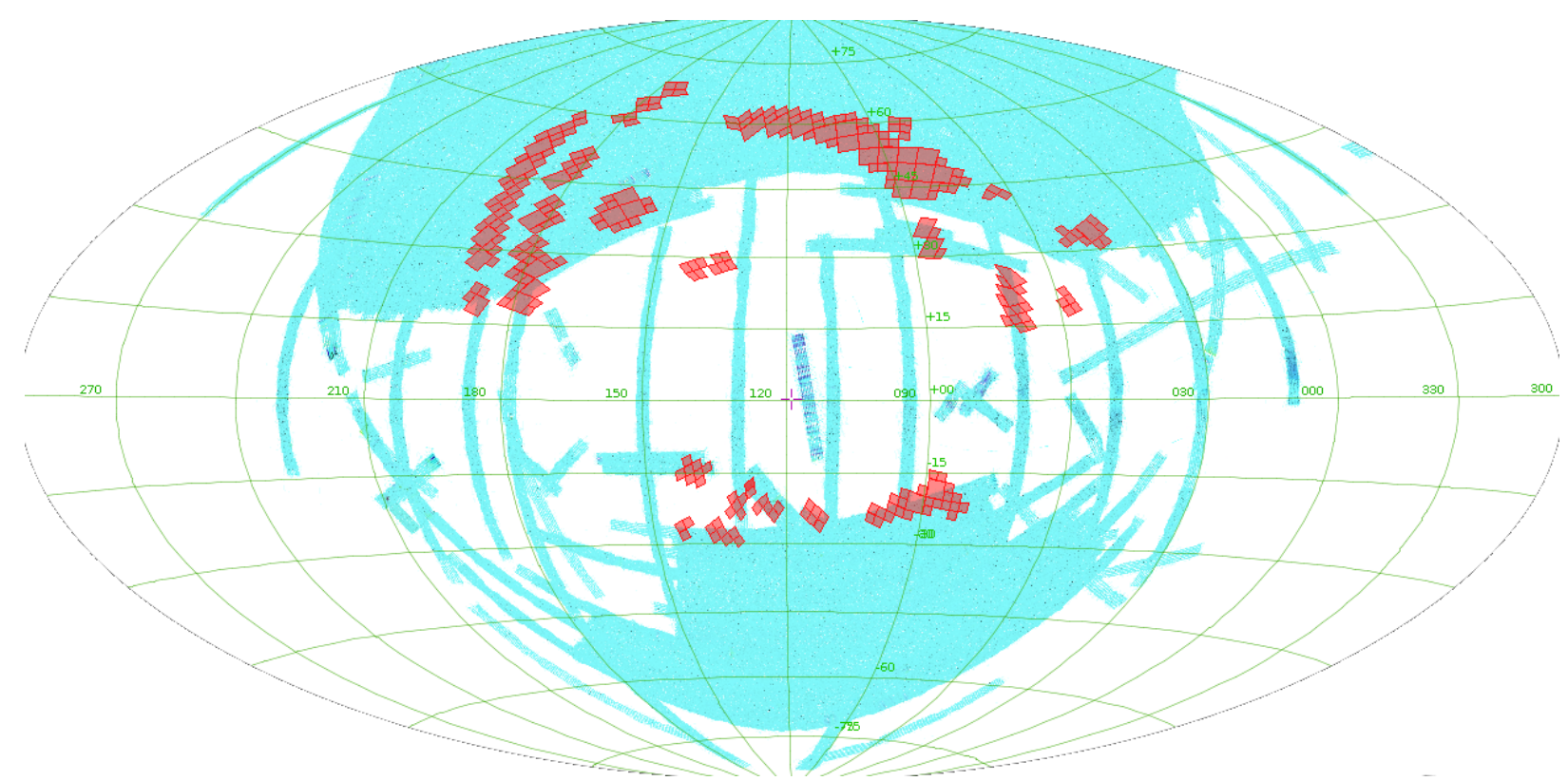

Fig. 1. Sky coverage of J-PLUS IDR201709 in Galactic coordinates (red). The SDSS DR9 footprint (blue) is plotted for comparison.

main source of UCD identifications is expected to be the Euclid surveys (Deacon 2018).

In this context, we plan to search for UCDs in Javalambre Photometric Local Universe Survey (J-PLUS ${ }^{1}$ ) and Javalambre Physics of the Accelerating Universe Astrophysical Survey $\left(\mathrm{J}_{-} \mathrm{PAS}^{2}\right)$, taking advantage of the Virtual Observatory ${ }^{3}$ (VO) capabilities. VO has proven to be an excellent methodology to identify and characterise cool objects (e.g. Aberasturi et al. 2011, 2014; Martín et al. 2013; Gálvez-Ortiz et al. 2017; Lodieu et al. 2017). In this paper we present the first search using the J-PLUS Internal Data Release catalogue, which covers $528 \mathrm{deg}^{2}$ ( $1 \%$ of the total sky area) in the blue and red optical bands.

The main advantage of J-PLUS compared to the previously cited surveys is its larger number of filters, which provide a denser sampling of the spectral energy distribution (SED) and allows a more accurate estimation of the effective temperature, which is a key parameter for classifying an object as a UCD. The fact that all photometric information comes from the same survey also minimises the risk of mismatching among surveys conducted at different epochs, in particular for the most interesting nearby sources with high proper motion.

This paper is organised as follows. In Sect. 2 we describe the J-PLUS survey. Section 3 is devoted to explaining the methodology that we have used to identify candidate ultracool dwarfs. In Sect. 4 we present some properties of our candidates. Finally, we summarise our work and present our conclusions in Sect. 5.

\section{J-PLUS}

J-PLUS is an ongoing multi-filter survey carried out with the Javalambre Auxiliary Survey Telescope (JAST/T80), a $0.83 \mathrm{~m}$ telescope installed at the Observatorio Astrofísico de Javalambre (OAJ) in Teruel, Spain. The survey uses the panoramic camera T80Cam that provides a large field of view of $1.4 \times 1.4 \mathrm{deg}^{2}$ with a pixel scale of $0.55 \operatorname{arcsec}_{\text {pixel }}{ }^{-1}$. J-PLUS was primarily conceived to perform the calibration tasks for the main J-

\footnotetext{
1 https://www.j-plus.es/

2 http://www.j-pas.org/

http://WWW . ivoa.net
}

PAS survey, observing the same regions of the sky $\left(\approx 8500 \mathrm{deg}^{2}\right)$. J-PAS (Benítez et al. 2014) is a photometric sky survey in 59 colours that will allow for the first time to map not only the positions of hundreds of millions of galaxies in the sky, but also their individual distances. This will provide the first complete 3D map of the Universe.

The J-PLUS filter system is composed of four broad- $(g$, $r, i$, and $z$ ), two intermediate- ( $u$ and J0861), and six narrowband (J0378,J0395,J0410,J0430,J0515, and J0660) filters optimised to provide an adequate sampling of the target SED in the optical range. The transmission curves as well as additional properties of the set of filters can be found at the Filter Profile Service maintained by the Spanish Virtual Observatory ${ }^{4}$. The expected limiting magnitude ( $3 \sigma$ in 3 arcsec aperture) is $\sim 21 \mathrm{mag}$, with $g$ and $r$ reaching $\sim 22 \mathrm{mag}$. J-PLUS magnitudes are given in the $\mathrm{AB}$ system.

The final survey parameters, scientific goals, and the technical requirements of the filter set are described in Cenarro et al. (2019). J-PLUS data have already been used in different research fields, such as the study of the Coma cluster (Jiménez-Teja et al. 2019), the analysis of the $\mathrm{H} \alpha$ emission in the nearby Universe (Logroño-García et al. 2019), the study of the M 15 globular cluster (Bonatto et al. 2019), the identification of new members in a galaxy cluster (Molino et al. 2019), the study of the stellar populations of several local galaxies (San Roman et al. 2019), or the study of the stellar and galactic morphological classification (López-Sanjuan et al. 2019).

\section{Analysis}

We queried the J-PLUS archive ${ }^{5}$, in particular the Internal Data Release (IDR201709), using the VO Asynchronous Queries option. IDR201709 comprises 264 J-PLUS pointings observed in the 12 optical bands described in Sect. 2, amounting to $528 \mathrm{deg}^{2}$ (Fig. 1). These pointings, available for the J-PLUS collaboration in September 2017, are a subset of the 511 pointings

\footnotetext{
4 https://bit.1y/20XuwNs

5 https://archive.cefca.es/catalogues
} 
that comprise the first J-PLUS data release, DR $1^{6}$, presented by Cenarro et al. (2019). Differences at $0.1 \mathrm{mag}$ level in the photometry of the same source between IDR201709 and DR1 can exist due to improvements in the reduction and the calibration processes made after September 2017. IDR201709 includes two types of catalogues: single catalogues, where the source detection and photometry were made on each band independently, and dual catalogues, where the detection and photometry were made using the $r$-band image as a reference. As UCDs emit most of their flux at longer wavelengths, we decided not to be linked to the $r$ band and instead used the single catalogues in our analysis. For this, we cross-matched the sources that were extracted from the images at different bands, assuming that sources separated by less than 1 arcsec are the same source.

\subsection{Photometric search}

We restricted our query to stellar sources. This was done using the class_star parameter of SExtractor (Bertin \& Arnouts 1996). Sources with class_star $>0.5$ in the $r$-band image are considered "stars" in the J-PLUS archive. In order to minimise the contamination by non-stellar sources, we were more restrictive and imposed class_star $>0.8$. We also required good photometric conditions (flag=0). An example of a typical query looks like this:

SELECT * FROM jplus.CalibratedMagABSingleObj,jplus.Filter WHERE jplus.Filter.FILTER_ID = jplus.CalibratedMagABSingleObj.FILTER_ID AND ALPHA_J2000 BETWEEN 145.0 AND 146.0

AND DELTA_J2000 BETWEEN 30.0 AND 33.0

AND CalibratedMagABSingleObj. FLAGS $=0$ A

AND CalibratedMagABSingleObj.CLASS_STAR $>0.8$

Figure 2 shows the distribution of the $i-z$ J-PLUS colours for the list of spectroscopically confirmed $\mathrm{M}$ dwarfs given by West et al. (2011) that is included in IDR201709. A list of 1257 stars was obtained from this cross-match (Table 1). For this subset, $i-z$ colour ranges from a mean value of $i-z \sim 0.3$ mag at subtype M0 V to $i-z \sim 1.3$ mag at subtype M9 V. We adopted a colour cut of $i-z>0.7 \mathrm{mag}$ as a criterion for identifying new UCDs. It includes almost all stars of subtype M7 V and later, at the expense of some contamination from earlier subtypes (M2$6 \mathrm{~V})$. To minimise this contamination as much as possible, we used VOSA ${ }^{7}$ (VO Sed Analyser; Bayo et al. 2008) to derive the effective temperature of the photometrically selected candidate UCDs (i.e. objects with $i-z>0.7 \mathrm{mag}$ ). VOSA is a tool developed by the Spanish Virtual Observatory and is designed to estimate physical parameters from the comparison of the observed target SED to different collections of theoretical models (see an illustrative example in Fig. 3).

Observational SEDs covering the optical and infrared range were built using the photometric catalogues available in VOSA. Then, SEDs were compared to the grid of BT-Settl CIFITS model atmospheres (Baraffe et al. 2015) to estimate effective temperatures. We assumed $\log g \geq 4.5$ and solar metallicity. GALEX photometry was not included in the SED because for M dwarfs, most of the flux in the GALEX bandpasses does not have a photospheric origin but is chromospheric (Shkolnik et al. 2011). Consequently, the flux observed at GALEX bands is systematically higher than the flux estimated from the photospheric models, which would have a clear impact on the SED fitting.

\footnotetext{
6 https://www.j-plus.es/datareleases/data_release_dr1

7 http://svo2.cab.inta-csic.es/theory/vosa/
}

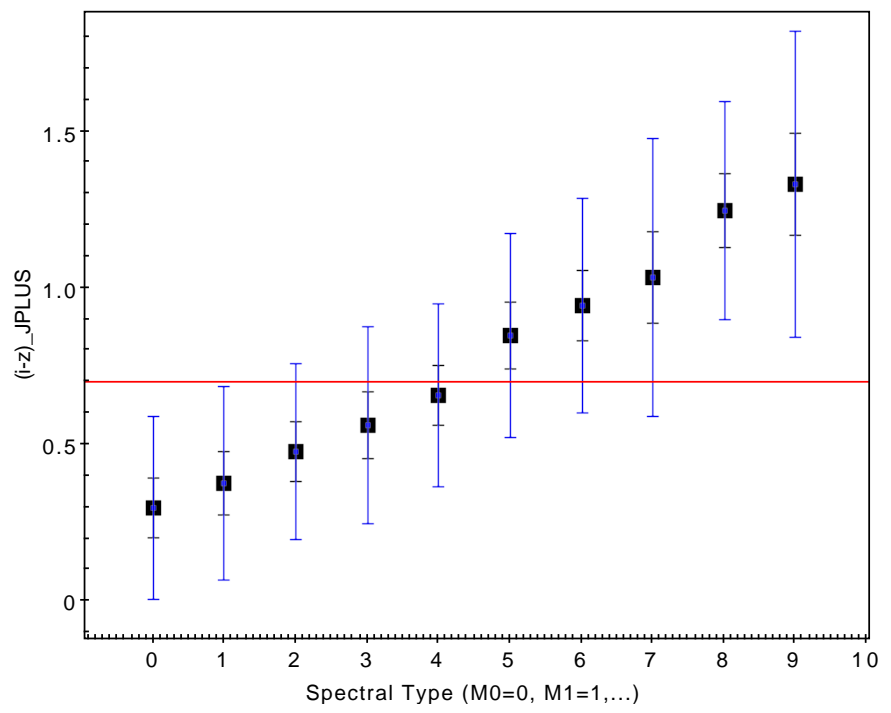

Fig. 2. Mean colour $i-z$ as a function of spectral type for the M dwarfs that are included in West et al. (2011) and with observations in J-PLUS. The red line represents the $i-z=0.7$ mag colour cut. Bars at $1 \sigma$ and $3 \sigma$ are also shown.

Table 1. Number of M dwarfs in West et al. (2011) with $i, z$ photometry in J-PLUS.

\begin{tabular}{lc}
\hline \hline Spectral type & Number of objects \\
\hline M0 V & 111 \\
M1 V & 79 \\
M2 V & 131 \\
M3 V & 136 \\
M4 V & 119 \\
M5 V & 92 \\
M6 V & 298 \\
M7 V & 239 \\
M8 V & 39 \\
M9 V & 13 \\
\hline
\end{tabular}

Extinction can play an important role in shaping the SED and therefore in the estimation of physical parameters. To account for this effect, we decided to leave extinction as a free parameter in the SED fitting process with values ranging from $A_{V}=0.0 \mathrm{mag}$ to $0.7 \mathrm{mag}$.

Using the 70840 spectroscopically confirmed M dwarfs available in West et al. (2011), we derived a relationship between spectral types and effective temperatures calculated using the VOSA and Baraffe et al. (2015) models (Table 2). According to this, we adopted for UCDs a conservative value of $T_{\text {eff }} \leq 2900 \mathrm{~K}$. With this criterion we may loose a small fraction of hotter UCDs, but at the same time, we avoid contamination from a significant number of M5-6 dwarfs.

The SEDs of the photometrically selected ultracool candidates with $T_{\text {eff }} \leq 2900 \mathrm{~K}$ were visually inspected to remove bad photometric points (i.e. points that clearly deviate from the theoretical SED) that could be affecting the quality of the SED fitting and therefore the estimation of the effective temperature. Moreover, we used the Aladin sky atlas (Bonnarel et al. 2000) to inspect the associated images and discard any problem related to blending or contamination by nearby sources.

The next step was to place our photometrically selected candidate UCDs in a colour-magnitude diagram. To do so, we 


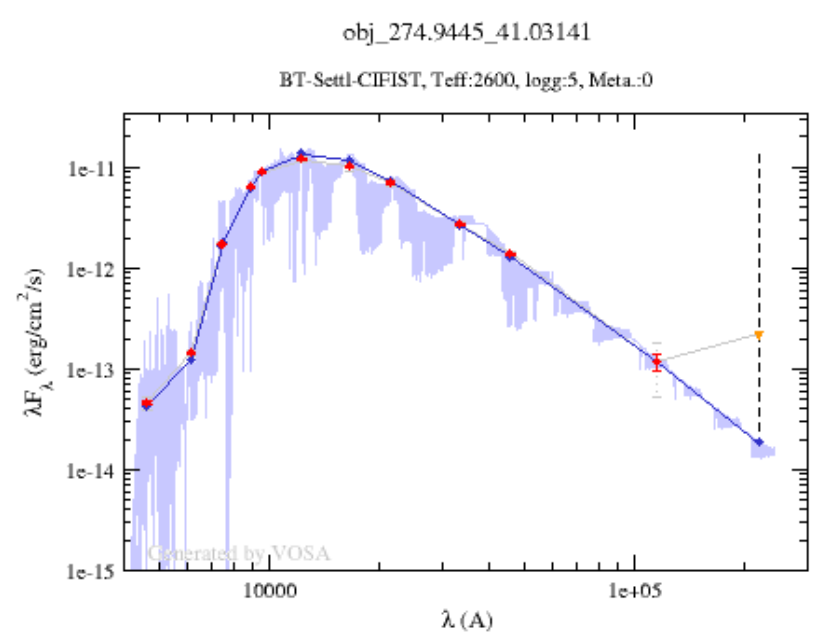

Fig. 3. Example of an SED fitting as generated by VOSA. The blue spectrum represents the theoretical model that fits best, while red dots represent the observed photometry. The inverted yellow triangle indicates that the photometric value corresponds to an upper limit. The vertical dashed line flags a possible excess in the SED.

Table 2. Mean effective temperatures calculated using VOSA and BTSettl-CIFITS models as a function of the spectral type given by West et al. (2011).

\begin{tabular}{lcc}
\hline \hline $\begin{array}{l}\text { Spectral } \\
\text { type }\end{array}$ & $\begin{array}{c}\text { Number of } \\
\text { objects }\end{array}$ & $\begin{array}{c}T_{\text {eff }} \\
(\mathrm{K})\end{array}$ \\
\hline M5 V & 3901 & $3210 \pm 210$ \\
M6 V & 5645 & $3060 \pm 110$ \\
M7 V & 5824 & $2960 \pm 150$ \\
M8 V & 1682 & $2710 \pm 160$ \\
M9 V & 891 & $2600 \pm 160$ \\
\hline
\end{tabular}

reproduced the diagram shown in Fig. 6 of Babusiaux et al. (2018) with all the Gaia DR2 objects at less than $100 \mathrm{pc}$. We kept only the candidates with relative errors of less than $10 \%$ in $G$ and $G_{\mathrm{RP}}$ and less than $20 \%$ in parallax. The absolute Gaia magnitude in the $G$ band for individual stars was estimated using

$M_{G}=G+5 \log \varpi+5$,

where $\varpi$ is the parallax in arcseconds. In our case, the inverse of the parallax is a reliable distance estimator because we kept only sources with relative errors in parallax lower than 20\% (Luri et al. 2018).

We also forced all Gaia counterparts to have a value of visibility_periods_used $>6$, which is the minimum value to accept a five-parameter astrometric solution for Gaia sources (Lindegren et al. 2018). This keyword indicates the number of groups of observations separated from other groups by a gap of at least four days (Babusiaux et al. 2018). After all these filtering processes, we ended up with 127 new candidate UCDs that have not previously been reported in the literature. Figure 4 shows the position of these sources in the CMD diagram. It confirms the dwarf and cool nature of all our candidates.

\subsection{Kinematic search}

Proper motions have been widely used in the literature to distinguish $\mathrm{M}$ dwarfs from other objects with similar colours but different kinematics, such as $\mathrm{M}$ giants, quasars, or distant red

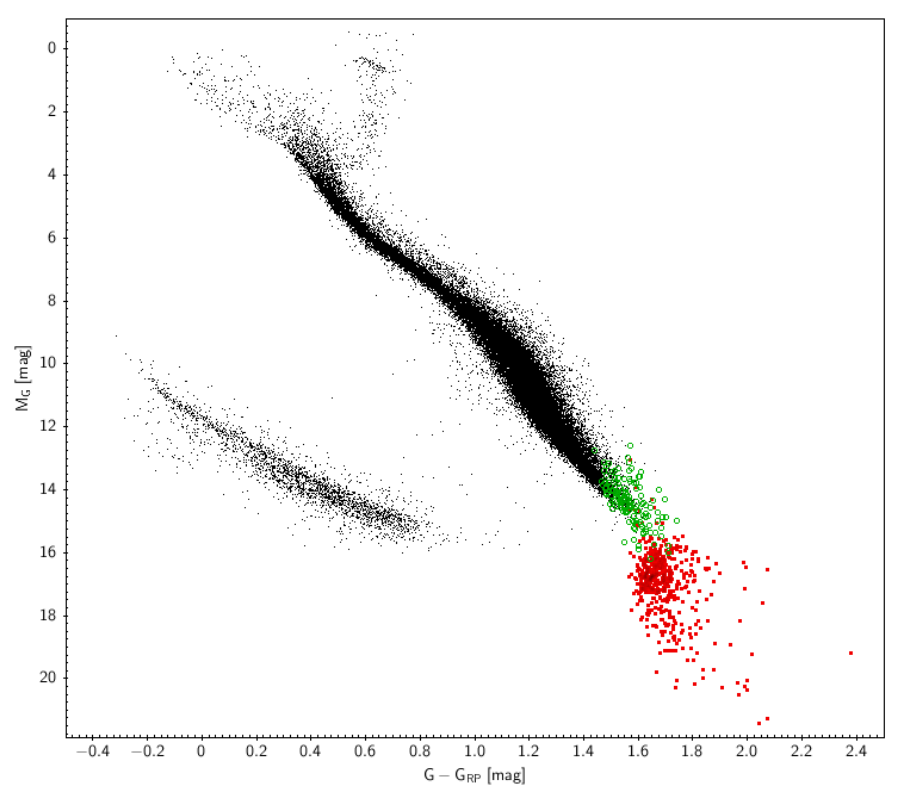

Fig. 4. Location of our photometrically selected candidate UCDs (open green circles) in a colour-magnitude diagram built using Gaia DR2 sources with parallaxes larger than 10 mas (black dots). $\mathrm{L}$ and $\mathrm{T}$ dwarfs identified in Smart et al. (2017) that have counterparts in Gaia DR2 are overplotted in red.

luminous galaxies (e.g. Theissen et al. 2016, 2017; Caballero et al. 2008). Moreover, proper motions are very useful to identify ultracool dwarfs with atypical colours due to anomalous values of gravity (Schmidt et al. 2010a) or metallicity (Bochanski et al. 2013; Zhang et al. 2018) that would be missed by colour cuts used in photometry-only searches.

The J-PLUS limiting magnitudes in the $i$ and $z$ band together with the UCD colour-distance relationship provided in Theissen et al. (2017) gives a maximum distance of about $400 \mathrm{pc}$ to find UCDs in the J-PLUS survey. This implies that UCDs seen by J-PLUS are at distances where proper motions are significant.

To define the proper motion cut between $M$ dwarfs and $M$ subgiants and giants, we took all objects classified in SIMBAD $^{8}$ (Wenger et al. 2000) as M subgiant or giant (luminosity classes IV, IV/III, and III) and with spectral type later than M5 (1 093 objects), and searched for their proper motions in Gaia DR2. Their normalised total proper motion distribution is compared in Fig. 5 with that of the 127 candidate UCDs identified in Sect. 3.1.

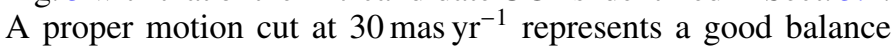
between giant contamination and dwarf completeness.

In the kinematic search we limited our query in J-PLUS to class_star $\geq 0.5$. Compared to the photometric search, we relaxed this condition from 0.8 to 0.5 based on two reasons. First, it increases the number of objects to be studied. Second, the contamination of non-stellar objects that are incorrectly flagged with class_star $>0.5$ can be easily removed using the proper motion information because extragalactic objects have negligible spatial motions. Sources fulfilling this condition were cross-matched with the Gaia DR2 catalogue to obtain the information on proper motions. A search radius of $10 \operatorname{arcsec}$ was used. If more than one counterpart existed in that radius, we took the nearest one. Of the 2620881 J-PLUS sources with Gaia DR2 counterparts, 64134 have total proper motions higher than 30 mas $r^{-1}$.

8 http://simbad.u-strasbg.fr/simbad/ 


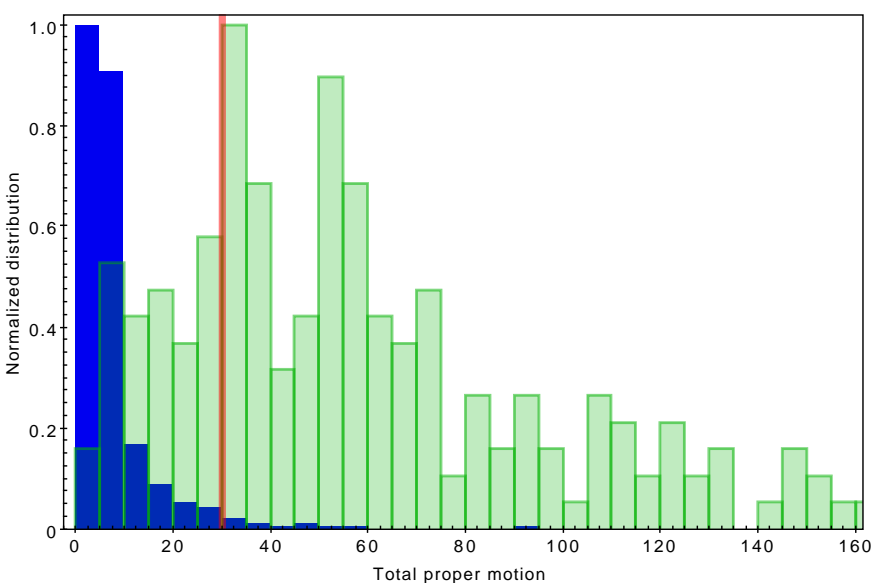

Fig. 5. Normalised distribution functions of the Gaia DR2 proper motions of the subgiants and giants available in SIMBAD with spectral type later than M5 (blue) and the 127 candidate UCDs identified in

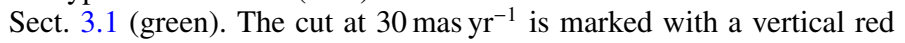
line.

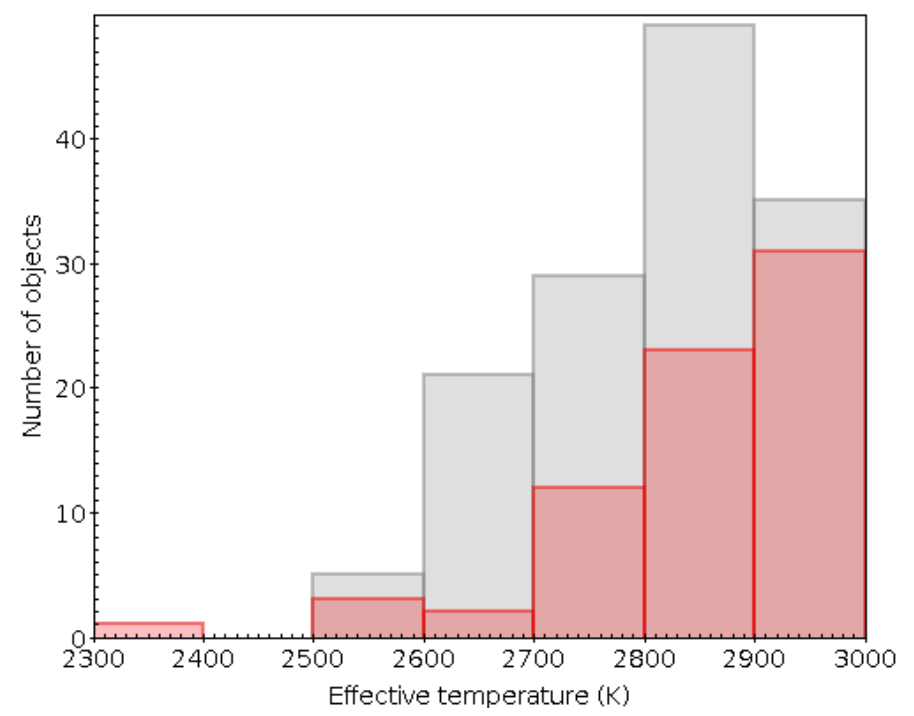

Fig. 6. $T_{\text {eff }}$ distribution of the candidate UCDs identified using photometry (grey) and proper motions (red). Most of our candidate UCDs $(\sim 65 \%)$ have $T_{\text {eff }}>2800 \mathrm{~K}$.

To remove hotter objects (spectral types earlier than M7) with high proper motions, we estimated effective temperatures for the 64134 objects using VOSA and followed a procedure similar to that described in Sect. 3.1. Furthermore, objects with Gaia counterparts with a visibility_periods_used $<6$ and astrometric_excess_noise $>1$ were removed. The latter is a Gaia parameter that quantifies the scatter of residuals in the astrometric solution. According to Lindegren et al. (2018), values above 1 will most likely correspond to artefacts. After this filtering, 60 new UCDs candidate were kept. These objects were not identified in the photometric search mainly because of two reasons: J-PLUS class_star $\leq 0.8$ or poor photometry in the J-PLUS $i$-, $z$-bands. The distribution of effective temperatures of our candidate UCDs (identified both using photometry and proper motions) are given in Fig. 6.

Figure 7 shows the position of the 60 kinematically-selected candidate UCDs in a reduced proper motion diagram:

$H_{G}=G+5 \log \mu+5$,

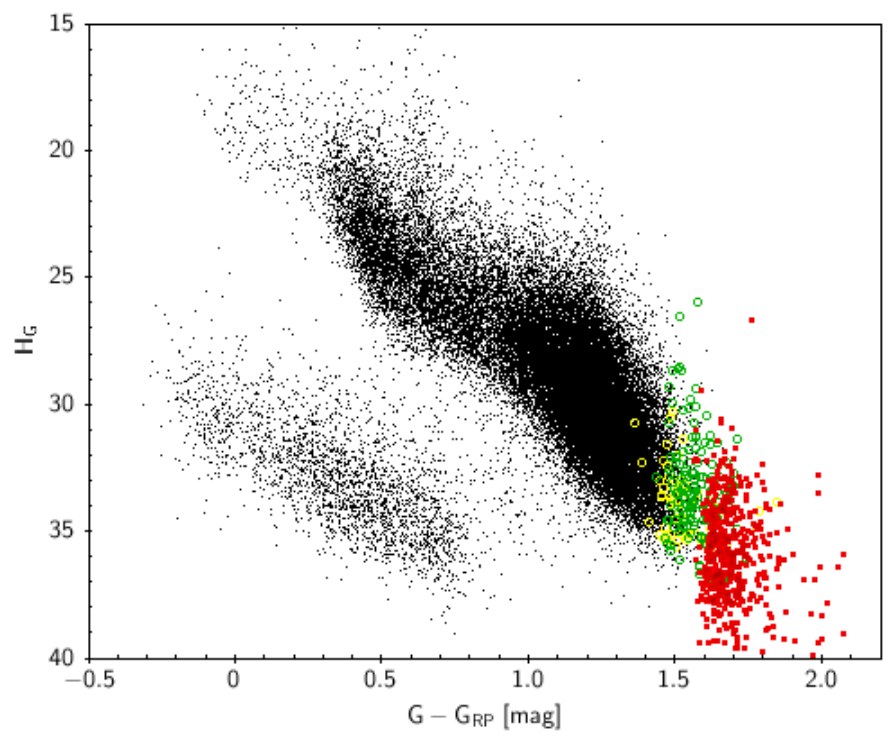

Fig. 7. Reduced proper motion - colour diagram. In black we plot the objects used in Fig. 4. Green open circles represent the 127 candidate UCDs identified in Sect. 3.1. Yellow open circles indicate the 60 kinematically selected candidate UCDs. L and T dwarfs from Smart et al. (2017) used in Fig. 4 are plotted as red squares.

where $G$ is the Gaia magnitude and $\mu$ is the total proper motion in mas $\mathrm{yr}^{-1}$. In this type of diagram, proper motion is used as a proxy for distance assuming that nearby objects will have higher proper motions. Just as in a Hertzsprung-Russell diagram, reduced proper motion diagrams have been shown to be excellent tools for segregating the various stellar populations (Dhital et al. 2010; Jiménez-Esteban et al. 2011, 2012; Zhang et al. 2013; Smart et al. 2017). All our candidates lie in the expected locus for ultracool dwarfs.

The approach adopted in this work for the kinematic search has two main limitations. First, we kept only objects with a min-

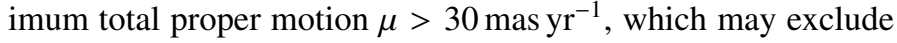
a non-negligible amount of nearby objects. This can be seen in Fig. 5, where we favour a low degree of contamination to completeness. Second, our procedure is based on Gaia proper motions. Gaia operates at optical wavelengths with a limiting magnitude $G \sim 21.4 \mathrm{mag}$, which makes it incomplete for UCDs, in particular for the reddest objects, as they emit predominantly in the infrared and are very faint in the Gaia bands (e.g. Smart et al. 2017). An alternative to this approach would be to estimate proper motions from the positions in optical and infrared surveys taken at different epochs, a methodology that is beyond the scope of this exploratory paper. Moreover, using tangential velocities, where information on distances and proper motions is combined, could be an alternative approach to separate dwarfs from giants. However, the number of Gaia DR2 parallaxes accurate enough to provide unbiased estimates of distances is significantly lower than the number of accurate proper motions (i.e. relative errors below $10 \%$ in both components). Using tangential velocities would result in the loss of $20-25 \%$ of our kinematically selected candidates, which led us to discard them from our kinematic search of UCDs.

A sample of the photometrically (127) and kinematically (60) selected candidate UCDs is given in Table 3, while the full list of candidates is available online at the SVO-JPLUS archive of ultracool dwarfs (see Appendix A). This service will be updated and maintained in the framework of the Spanish Virtual 
Table 3. New candidate ultracool dwarfs.

\begin{tabular}{lccccrrrrr}
\hline \hline $\begin{array}{l}\alpha \\
(\mathrm{ICRS}, \mathrm{deg})\end{array}$ & $\begin{array}{c}\delta \\
(\mathrm{ICRS}, \mathrm{deg})\end{array}$ & $\begin{array}{c}T_{\mathrm{eff}} \\
(\mathrm{K})\end{array}$ & $\begin{array}{c}\mathrm{G} \\
(\mathrm{mag})\end{array}$ & $\begin{array}{c}\varpi \\
(\mathrm{mas})\end{array}$ & $\begin{array}{c}\mu_{\alpha} \cos \delta \\
\left(\mathrm{mas} \mathrm{yr}^{-1}\right)\end{array}$ & $\begin{array}{c}\mu_{\delta} \\
\left(\mathrm{mas} \mathrm{yr}^{-1}\right)\end{array}$ & Search & $\begin{array}{c}i \\
(\mathrm{mag})\end{array}$ & $\begin{array}{c}z \\
(\mathrm{mag})\end{array}$ \\
\hline 21.70712 & 33.44938 & 2600 & 20.94 & 11.18 & 7.36 & -51.33 & $\mathrm{P}$ & 20.19 & 18.69 \\
22.16372 & 33.30222 & 2600 & 18.44 & 18.93 & 77.34 & -99.44 & $\mathrm{P}$ & 17.81 & 16.33 \\
23.15882 & 39.59608 & 2800 & 20.58 & 3.41 & -54.42 & -21.40 & $\mathrm{~K}$ & & \\
27.63937 & 33.73132 & 2900 & 19.84 & 3.79 & 2.44 & -39.94 & $\mathrm{P}$ & 19.34 & 18.11 \\
28.13097 & 33.83401 & 2600 & 19.69 & 11.93 & 58.33 & -46.75 & $\mathrm{P}$ & 19.11 & 17.67
\end{tabular}

Notes. Equatorial coordinates, $G$ magnitudes, parallaxes, and proper motions are from Gaia DR2, effective temperatures are estimated with VOSA and $i, z$ magnitudes are from J-PLUS. The column "Search" indicates whether the source was discovered in the photometric (P) or in the kinematic (K) search. The full table is available at the SVO-JPLUS archive of ultracool dwarfs (see Appendix A).

Observatory $^{9}$. According to VOSA criteria, based on the slope of the SED and the flux difference between the observed and theoretical SEDs (see the VOSA help ${ }^{10}$ for a detailed description on how VOSA handles the infrared excess), none of the candidate UCDs shows excess in the infrared that could be ascribed to circumstellar material or to a close ultracool companion. This result is in agreement with the ratio of $\mathrm{M}$ dwarfs with infrared excess given by Theissen \& West (2014), estimated to be one object in about every 400 stars.

\subsection{Known ultracool dwarfs}

In this section we assess the fraction of known UCDs that have been recovered using our methodology. In particular, we searched for UCDs in two catalogues: SIMBAD and Smart et al. (2017).

Using the SIMBAD TAP service ${ }^{11}$, we chose from the more than 9 million available objects those with spectral types M7 V, M8 V, M9 V. A total of 9894 objects fulfilled these conditions.

To know how many of them lie in the region of the sky covered by IDR201709, we took advantage of the Multi-Object Coverage $^{12}$ VO standard and Aladin. A total of 588 objects lie in the region of the sky observed in IDR201709. A query to the archive for these 588 objects returned 368 objects with J-PLUS data. We repeated the same methodology for Smart et al. (2017). Only 53 out of the 1885 objects included in the catalogue lie in the J-PLUS field, 7 of them with observations in the J-PLUS archive. The scarcity of J-PLUS counterparts can be explained by the intrinsic faintness of these objects, which are typically beyond the IDR201709 limiting magnitude.

The efficiency of our search was estimated using the falsenegative rate (number of known UCDs that were not rediscovered in our search). All SIMBAD sources (368) were found, while only four of the seven objects included in Smart et al. (2017) with J-PLUS observations were recovered, the remaining three have a poor SED fitting that prevented us from deriving their effective temperatures. These $372(368+4)$ objects were not included in our list of $187(127+60)$ objects as they have already been reported in the literature. This gives a total of 559 UCDs identified in this paper. The 187 new candidate UCDs compared to the 9894 UCDs already included in SIM$\mathrm{BAD}$ represents $2 \%$ of new discoveries. However, if we restrict our analysis to the area of the sky covered by J-PLUS DR1

\footnotetext{
9 http://svo.cab.inta-csic.es

10 https://bit.1y/2yzwyIF

11 http://simbad.u-strasbg.fr: 80/simbad/sim-tap

12 http://ivoa.net/documents/MOC/index.html
}

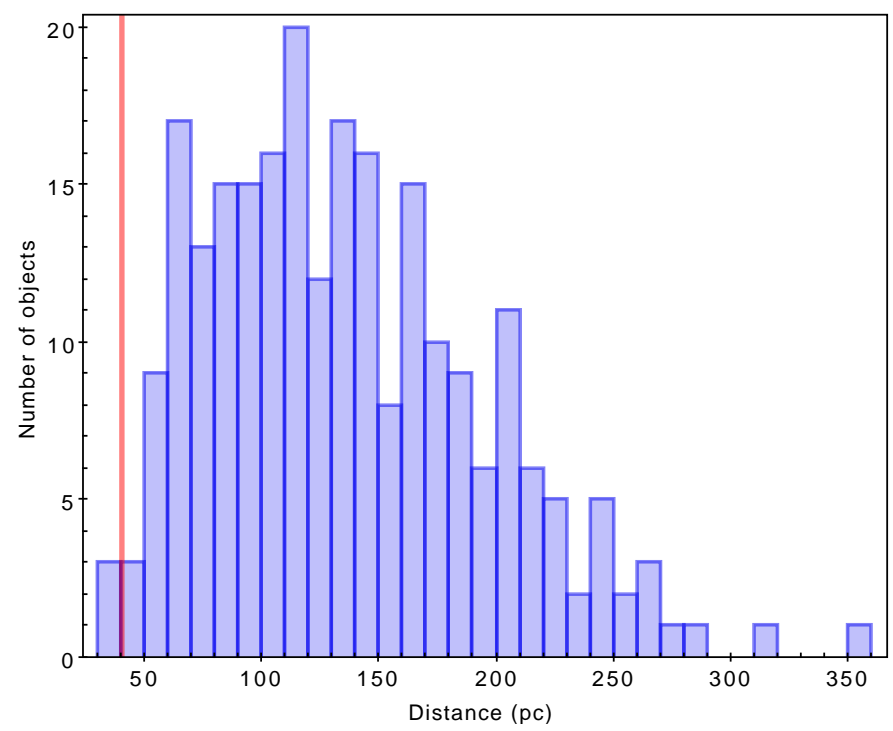

Fig. 8. Distance distribution for our candidate UCDs with good Gaia DR2 parallaxes $(\delta \varpi / \varpi<20 \%)$. The red vertical line indicates $d=$ $40 \mathrm{pc}$. The mean value of the distribution is $130 \mathrm{pc}$, with the closest and farthest objects at $32 \mathrm{pc}$ and $352 \mathrm{pc}$.

$\left(528 \mathrm{deg}^{2}\right)$, the increase in the number of known UCDs is about $50 \%$ (187/372). This ratio of new discoveries is not evenly distributed with spectral types, it is higher $(\sim 5 \%)$ for the cooler (>M8) objects. Extrapolating these results to the predicted sky coverage at the end of the J-PLUS survey $\left(8500 \mathrm{deg}^{2}\right)$, we would expect to discoverabout 3000-3500 new UCDs, which represents one-third of the UCDs that are currently included in SIMBAD. These results confirm the robustness of our methodology in discovering ultracool dwarfs.

\section{Results and discussion}

\subsection{Distances}

To avoid biases due to large errors in parallax (Astraatmadja \& Bailer-Jones 2016), distances were estimated only if the relative error in parallax was smaller than $20 \%$. As in Sect. 3.1, we used the inverse of the parallaxes given in Gaia DR2. The distribution of distances for our candidate UCDs is shown in Fig. 8.

Because of the comprehensive search done in the past for nearby UCDs, the great majority of the new candidate UCDs presented in this paper are at distances greater than $40 \mathrm{pc}$, which is interesting as reference for future wide and deep surveys such 
Table 4. Newly identified nearby $(d<40 \mathrm{pc})$ candidate UCDs.

\begin{tabular}{lccccccc}
\hline \hline $\begin{array}{l}\text { Gaia ID } \\
\text { DR2 }\end{array}$ & $\begin{array}{c}\alpha \\
(\mathrm{deg})\end{array}$ & $\begin{array}{c}\delta \\
(\mathrm{deg})\end{array}$ & $\begin{array}{c}G \\
(\mathrm{mag})\end{array}$ & $\begin{array}{c}T_{\mathrm{eff}} \\
(\mathrm{K})\end{array}$ & $\begin{array}{c}\mu_{\alpha} \cos \delta \\
\left(\mathrm{mas} \mathrm{yr}^{-1}\right)\end{array}$ & $\begin{array}{c}\mu_{\delta} \\
\left(\mathrm{mas} \mathrm{yr}^{-1}\right)\end{array}$ & $\begin{array}{c}d \\
(\mathrm{pc})\end{array}$ \\
\hline 1902388491693623680 & 338.44505 & 33.99409 & 17.57 & 2900 & $254.44 \pm 0.25$ & $104.42 \pm 0.26$ & $32.45 \pm 0.15$ \\
2109889524381399040 & 274.94447 & 41.03144 & 17.89 & 2700 & $21.30 \pm 0.23$ & $-250.82 \pm 0.24$ & $39.60 \pm 0.19$ \\
1596590634148306944 & 236.27034 & 52.23673 & 17.16 & 2900 & $57.26 \pm 0.18$ & $86.54 \pm 0.18$ & $39.98 \pm 0.15$ \\
\hline
\end{tabular}

Notes. Coordinates are from Gaia DR2. We adopted the step of the BT-Settl CIFITS grid of models (100 K) as the error in effective temperatures.

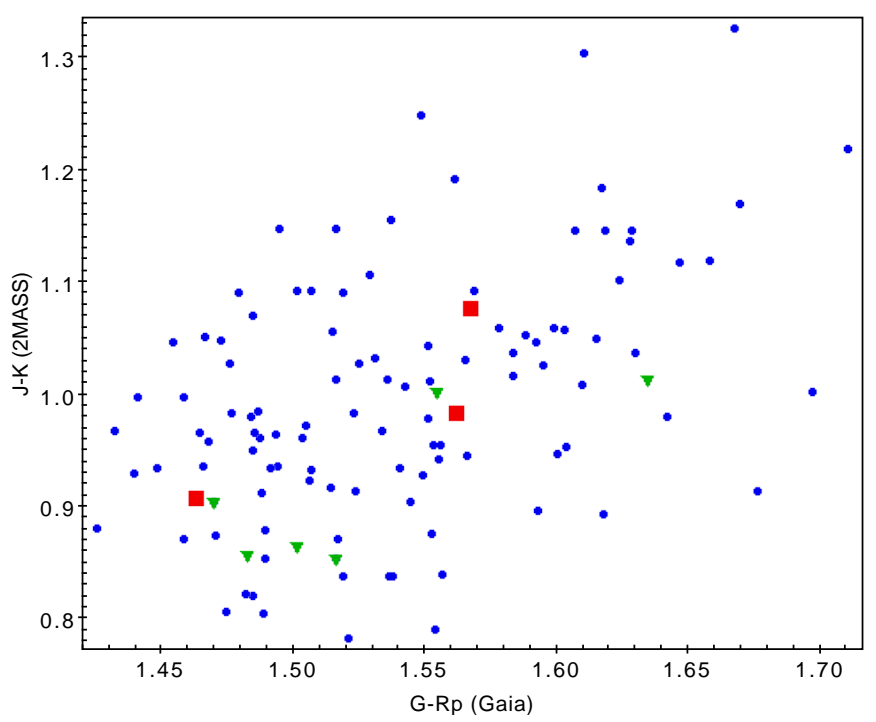

Fig. 9. $J-K_{s}$ vs. $G-R p$ colour-colour diagram of our candidate UCDs with good 2MASS photometry (Qflg=A) in $J$ and $K_{s}$ bands. Red squares represent our three candidate UCDs at distances $d<40 \mathrm{pc}$. Inverted green triangles indicate objects with tangential velocities $V_{\tan }>$ $100 \mathrm{~km} \mathrm{~s}^{-1}$

as Euclid (Racca et al. 2018). However, there are three objects at less than $40 \mathrm{pc}$ that were not detected in previous searches, which indicates that there is still room to discover new nearby UCDs even with small telescopes such as JAST/T80. We list the details of these three objects in Table 4. Based on the effective temperatures calculated with VOSA and the $T_{\text {eff }}-$ spectral type relationship given in Table 2, we estimated a photometric spectral types of $\mathbf{M 7}$ for $T_{\text {eff }} \sim 2900 \mathrm{~K}$ and $\mathbf{M 8}$ for $T_{\text {eff }} \sim 2700 \mathrm{~K}$.

Ultracool dwarfs may be missed by photometric surveys because their $J-K_{S}$ colours are unusual for their spectral types. Red $J-K_{s}$ outliers often exhibit distinctive low-gravity features, indicating that these objects are young and still collapsing. On the other hand, blue outliers show strong $\mathrm{H}_{2} \mathrm{O}$ and weak $\mathrm{CO}$ features in their infrared spectra, possibly associated with high gravity and old age (Burgasser et al. 2008; Schmidt et al. 2010b; Zhang et al. 2017). A paradigmatic example of these outliers is SDSS J141624.09+134826.7, an exceptionally blue L dwarf within $10 \mathrm{pc}$ of the Sun (Bowler et al. 2010; Schmidt et al. 2010a). Nevertheless, as shown in Fig. 9, this was not the case of our three candidate UCDs, which lie in the region of typical colours.

\subsection{Tangential velocities}

Kinematics is a good proxy to obtain information on the ages of UCDs as old stars belong to the thick disc and therefore have higher velocities than young stars, which belong to the thin disc (Montes et al. 2001; Zuckerman \& Song 2004).

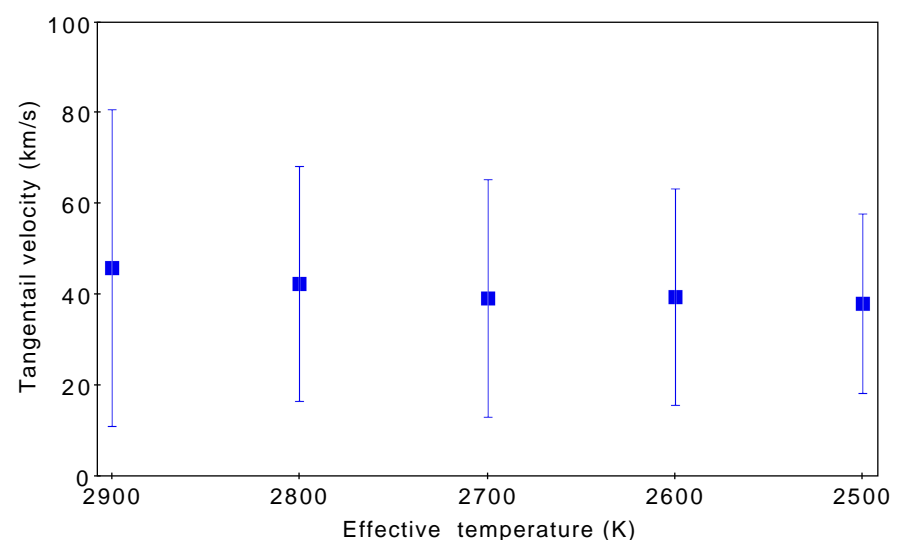

Fig. 10. Mean tangential velocities of our candidate UCDs. Error bars represent the standard deviation.

The lack of radial velocities for our ultracool candidates prevents us from making a three-dimensional kinematic analysis. Instead, by combining Gaia DR2 proper motions and distances, we used tangential velocities, defined as $V_{\tan }=4.74 \mu d$, where $V_{\tan }$ is given in $\mathrm{km} \mathrm{s}^{-1}, \mu$ is the total proper motion in $\operatorname{arcsec} \mathrm{yr}^{-1}$ and $d$ is the distance in pc.

Figure 10 shows the distribution of the mean tangential velocities with the effective temperature. The discretisation seen in the values of $T_{\mathrm{eff}}$ is a consequence of the step adopted in the CIFITS grid of models. The lack of correlation between tangential velocities and effective temperature indicates that our candidates represent a single kinematic population, regardless of the spectral type.

Figure 11 shows the distribution of tangential velocities. Six objects have $V_{\tan }>100 \mathrm{~km} \mathrm{~s}^{-1}$. Even when we include these objects, we obtain a median value of $V_{\tan }=34 \mathrm{~km} \mathrm{~s}^{-1}$ and a dispersion of $\sigma=26 \mathrm{~km} \mathrm{~s}^{-1}$, which coincides very well with previous determinations for ultracool dwarfs (Faherty et al. 2009).

These six high-velocity objects do not show unusual colours for their spectral type (Fig. 9). Adopting $V_{\tan }=180 \mathrm{~km} \mathrm{~s}^{-1}$ as the boundary between disc and halo objects (Sesar et al. 2008), we conclude that all our UCD candidates correspond to thindisc objects, with the six high-velocity objects being potential members of the thick disc.

\section{Conclusions}

Using a Virtual Observatory methodology, we have produced a catalogue of 187 new candidate ultracool dwarfs using J-PLUS IDR201709. Our goal was not to construct a complete catalogue. Rather, we attempted to build a catalogue of bona fide UCDs and to test a search methodology that could be used for new J-PLUS releases and, what is more interesting, for the deeper J-PAS survey, for which first light is scheduled in 2019. J-PAS, with its contiguous system of 54 narrow-band filters, will provide an 


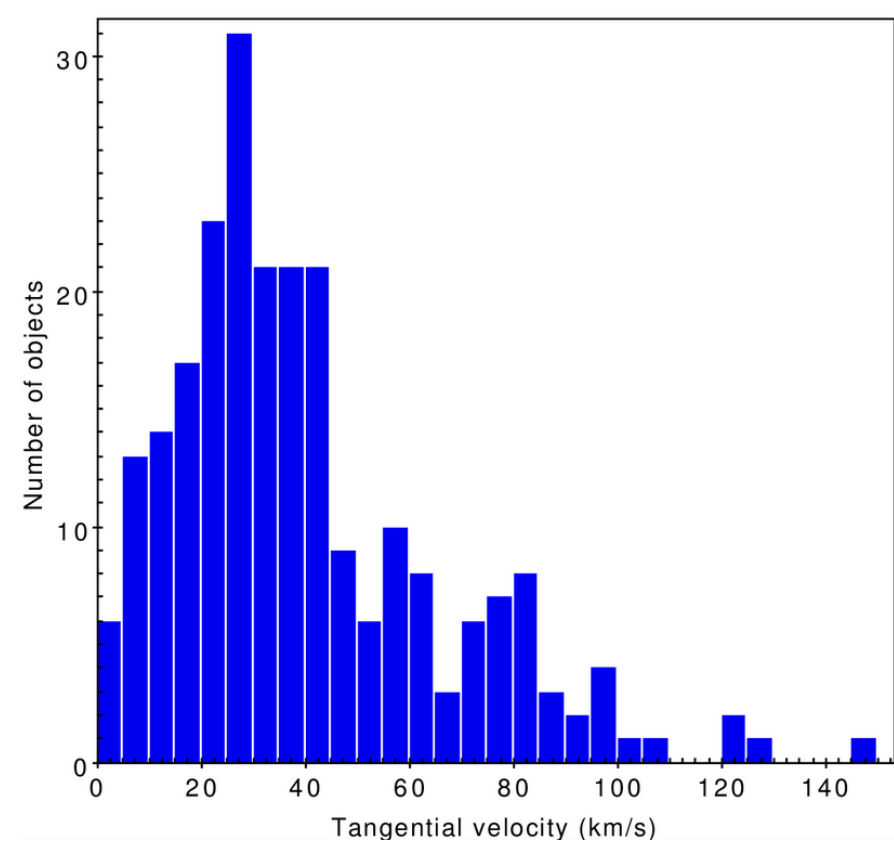

Fig. 11. Histogram of tangential velocities of our candidate UCDs.

unprecedented photometric coverage in the optical range, allowing more accurate determinations of the effective temperatures and probably a direct confirmation of the true UCD nature of our objects without the need for external spectroscopic follow-up.

The use of a dual methodology based on photometry and proper motions tends to minimise the drawbacks and biases associated with the search of ultracool objects: photometric-only selected samples may leave out peculiar UCDs that do not follow the canonical trend in colour-colour diagrams, while proper motion searches may ignore objects with low values of projected velocity in the plane of the sky. The high success ratio in recovering known UCDs (>95\%) demonstrates the robustness of our procedure.

With the help of the VOSA Virtual Observatory tool, we estimated effective temperatures for our candidate UCDs. They range from $\mathrm{M} 7 \mathrm{~V}$ to $\mathrm{LO} \mathrm{V}$, and the great majority of the objects have M7-8 V spectral types. Distances were computed using Gaia parallaxes with relative errors smaller than $20 \%$. A mean value of $130 \mathrm{pc}$ was obtained, with the closest and farthest objects at $32 \mathrm{pc}$ and $352 \mathrm{pc}$, respectively. We also identified three new systems with distances $d<40 \mathrm{pc}$.

Analysis on tangential velocities concluded that our candidate UCDs are consistent with the Galactic thin-disc population. However, six objects lie at the tail ${ }^{13}$ end of the velocity distribution $^{14}$ and are likely part of an older Galactic population.

Acknowledgements. We thank our referee, Zenghua Zhang, for his constructive suggestions. We warmly thank the J-PLUS collaboration, in particular, $\mathrm{S}$ Akras, A. Alvarez, P. Coelho, and V. Placco for constructive comments that helped to improve the presentation of the results. We acknowledge use of the ADS bibliographic services. This research has made use of the Aladin sky atlas developed at CDS, Strasbourg Observatory, France. This publication makes use of VOSA, developed under the Spanish Virtual Observatory project supported from the Spanish MINECO through grant AyA2017-84089. This work has made use of data from the European Space Agency (ESA) mission Gaia, processed by the Gaia Data Processing and Analysis Consortium (DPAC). We also extensively made use of the Vizier and SIMBAD services, both operated at CDS, Strasbourg, France. Funding for the J-PLUS Project has been provided

13 https://wwW. cosmos. esa.int/gaia

14 https://wwW. cosmos. esa.int/web/gaia/dpac/consortium by the Governments of Spain and Aragón through the Fondo de Inversiones de Teruel, the Spanish Ministry of Economy and Competitiveness (MINECO; under grants AYA2015-66211-C2-1-P, AYA2015-66211-C2-2, AyA2012-30789 and ICTS-2009-14), and European FEDER funding (FCDD10-4E-867, FCDD134E-2685). The Brazilian agencies FAPERJ and FAPESP as well as the National Observatory of Brazil have also contributed to this project. RAD acknowledges support from CNPq through BP grant 312307/2015-2, CSIC through grant COOPB20263, FINEP grants REF. R 1217/13 - 01.13.0279.00 and REF $0859 / 10-01.10 .0663 .00$ for hardware support for the J-PLUS project through the National Observatory of Brazil. E. L. Martín acknowledges support from project AYA2015-69350-C3-1-P. J. A. Hernandez-Jimenez thanks to Brazilian institution CNPq (project 150237/2017-0) and Chilean institution CONICYT through Programa de Astronomía, Fondo ALMA-CONICYT 2017 (project 31170038). R. Lopes was partially supported by the Brazilian agency CNPq (PQ 302037/2015-2 and PDE 200289/2017-9). F. J. E. acknowledges financial support from the ASTERICS project (ID:653477, H2020-EU.1.4.1.1. Developing new world-class research infrastructures). This work is based on observations made with the JAST/ T80 telescope at the Observatorio Astrofísico de Javalambre (OAJ) in Teruel, owned, managed and operated by the Centro de Estudios de Física del Cosmos de Aragón. We thank the OAJ Data Processing and Archiving Unit (UPAD) for reducing and calibrating the OAJ data used in this work.

\section{References}

Aberasturi, M., Solano, E., \& Martín, E. L. 2011, A\&A, 534, L7

Aberasturi, M., Caballero, J. A., Montesinos, B., et al. 2014, AJ, 148, 36 Anglada-Escudé, G., Amado, P. J., Barnes, J., et al. 2016, Nature, 536, 437 Astraatmadja, T. L., \& Bailer-Jones, C. A. L. 2016, ApJ, 833, 119 Babusiaux, C., van Leeuwen, F., Barstow, M. A., et al. 2018, A\&A, 616, A10 Baraffe, I., Homeier, D., Allard, F., \& Chabrier, G. 2015, A\&A, 577, A42 Bayo, A., Rodrigo, C., Barrado, Y., \& Navascués, D. 2008, A\&A, 492, 277 Benítez, N., Dupke, R., Moles, M., et al. 2014, ArXiv e-prints [arXiv:1403.5237]

Bertin, E., \& Arnouts, S. 1996, A\&AS, 117, 393

Bochanski, J. J., Savcheva, A., West, A. A., \& Hawley, S. L. 2013, AJ, 145, 40

Bonatto, C., Chies-Santos, A. L., Coelho, P. R. T., et al. 2019, A\&A, 622, A179

Bonnarel, F., Fernique, P., Bienaymé, O., et al. 2000, A\&AS, 143, 33

Bowler, B. P., Liu, M. C., \& Dupuy, T. J. 2010, ApJ, 710, 45

Burgasser, A. J., Looper, D. L., Kirkpatrick, J. D., Cruz, K. L., \& Swift, B. J. 2008, ApJ, 674, 451

Caballero, J. A. 2018, Geosciences, 8, 362

Caballero, J. A., Burgasser, A. J., \& Klement, R. 2008, A\&A, 488, 181

Cenarro, A. J., Moles, M., Cristóbal-Hornillos, D., et al. 2019, A\&A, 622, A176

Chabrier, G. 2003, PASP, 115, 763

Chabrier, G., \& Baraffe, I. 2000, ARA\&A, 38, 337

Cross, N. J. G., Collins, R. S., Mann, R. G., et al. 2012, A\&A, 548, A119

Deacon, N. R. 2018, MNRAS, 481, 447

Dhital, S., West, A. A., Stassun, K. G., \& Bochanski, J. J. 2010, AJ, 139, 2566

Dressing, C. D., \& Charbonneau, D. 2015, ApJ, 807, 45

Epchtein, N., Deul, E., Derriere, S., et al. 1999, A\&A, 349, 236

Faherty, J. K., Burgasser, A. J., Cruz, K. L., et al. 2009, AJ, 137, 1

Faherty, J. K., Rice, E. L., Cruz, K. L., Mamajek, E. E., \& Núñez, A. 2013, AJ, 145,2

Gálvez-Ortiz, M. C., Solano, E., Lodieu, N., \& Aberasturi, M. 2017, MNRAS, 466, 2983

Gillon, M., Jehin, E., Lederer, S. M., et al. 2016, Nature, 533, 221

Gillon, M., Triaud, A. H. M. J., Demory, B.-O., et al. 2017, Nature, 542, 456

Helling, C., Ackerman, A., Allard, F., et al. 2008, MNRAS, 391, 1854

Henry, T. J., Jao, W.-C., Subasavage, J. P., et al. 2006, AJ, 132, 2360

Jiménez-Esteban, F. M., Caballero, J. A., \& Solano, E. 2011, A\&A, 525, A29

Jiménez-Esteban, F. M., Caballero, J. A., Dorda, R., Miles-Páez, P. A., \& Solano, E. 2012, A\&A, 539, A86

Jiménez-Teja, Y., Dupke, R.A., \& Lopes de Oliveira, R. 2019, A\&A, 622, A183

Jones, H. R. A., \& Tsuji, T. 1997, ApJ, 480, L39

Kroupa, P. 2001, MNRAS, 322, 231

Lawrence, A., Warren, S. J., Almaini, O., et al. 2007, MNRAS, 379, 1599

Lindegren, L., Hernández, J., Bombrun, A., et al. 2018, A\&A, 616, A2

Lodieu, N., Espinoza Contreras, M., Zapatero Osorio, M. R., et al. 2017, A\&A, 598, A92

Logroño-García, R., Vilella-Rojo, G., López-Sanjuan, C., et al. 2019, A\&A, 622, A180

López-Sanjuan, C., Vázquez Ramió, H., Varela, J., et al. 2019, A\&A, 622, A177 Luri, X., Brown, A. G. A., Sarro, L. M., et al. 2018, A\&A, 616, A9

Martín, E. L., Cabrera, J., Martioli, E., Solano, E., \& Tata, R. 2013, A\&A, 555, A108 
Molino, A., Costa-Duarte, M. V., \& Mendes de Oliveira, C. 2019, A\&A, 622, A178

Montes, D., López-Santiago, J., Gálvez, M. C., et al. 2001, MNRAS, 328, 45

Racca, G., Laureijs, R., \& Mellier, Y. 2018, in 42nd COSPAR Scientific Assembly, E1.16-3-18, COSPAR Meeting, 42

Ribas, I., Tuomi, M., Reiners, A., et al. 2018, Nature, 563, 365

San Roman, I., Sánchez-Blázquez, P., Cenarro, A. J., et al. 2019, A\&A, 622, A181

Schmidt, S. J., West, A. A., Burgasser, A. J., Bochanski, J. J., \& Hawley, S. L. 2010a, AJ, 139, 1045

Schmidt, S. J., West, A. A., Hawley, S. L., \& Pineda, J. S. 2010b, AJ, 139, 1808

Sesar, B., Ivezić, Ž., \& Jurić, M. 2008, ApJ, 689, 1244

Shkolnik, E. L., Liu, M. C., Reid, I. N., Dupuy, T., \& Weinberger, A. J. 2011, ApJ, 727, 6

Skrutskie, M. F., Cutri, R. M., Stiening, R., et al. 2006, AJ, 131, 1163

Smart, R. L., Marocco, F., Caballero, J. A., et al. 2017, MNRAS, 469, 401

Theissen, C. A., \& West, A. A. 2014, ApJ, 794, 146

Theissen, C. A., West, A. A., \& Dhital, S. 2016, AJ, 151, 41

Theissen, C. A., West, A. A., Shippee, G., Burgasser, A. J., \& Schmidt, S. J. 2017, AJ, 153, 92

Wenger, M., Ochsenbein, F., Egret, D., et al. 2000, A\&AS, 143, 9

West, A. A., Morgan, D. P., Bochanski, J. J., et al. 2011, AJ, 141, 97

Wright, E. L., Eisenhardt, P. R. M., Mainzer, A. K., et al. 2010, AJ, 140, 1868

York, D.G., Adelman, J., \& Anderson John, E.J. 2000, AJ, 120, 1579

Zhang, Z. H., Pinfield, D. J., Burningham, B., et al. 2013, MNRAS, 434, 1005

Zhang, Z. H., Pinfield, D. J., Gálvez-Ortiz, M. C., et al. 2017, MNRAS, 464, 3040

Zhang, Z. H., Galvez-Ortiz, M. C., Pinfield, D. J., et al. 2018, MNRAS, 480, 5447

Zhang, Z. H., Burgasser, A. J., Gálvez-Ortiz, M. C., et al. 2019, MNRAS, 486 1260

Zuckerman, B., \& Song, I. 2004, ARA\&A, 42, 685

1 Departamento de Astrofísica, Centro de Astrobiología (CSICINTA), ESAC Campus, Camino Bajo del Castillo s/n, 28692 Villanueva de la Cañada, Madrid, Spain e-mail: esm@cab.inta-csic.es

2 Spanish Virtual Observatory, Spain

3 Departmento de Astrofísica, Centro de Astrobiología (CSIC-INTA), Carretera de Torrejón a Ajalvir km 4, 28850 Torrejón de Ardoz, Madrid, Spain

4 Centro de Estudios de Física del Cosmos de Aragón (CEFCA), Plaza San Juan 1, 44001 Teruel, Spain

5 Donostia International Physics Centre (DIPC), Paseo Manuel de Lardizabal 4, 20018 Donostia-San Sebastián, Spain

6 IKERBASQUE, Basque Foundation for Science, 48013 Bilbao, Spain

7 Observatorio Nacional - MCTIC (ON), Rua General José Cristino, 77, São Cristóvão, 20921-400 Rio de Janeiro, Brazil
${ }^{8}$ Centro de Estudios de Física del Cosmos de Aragón (CEFCA) Unidad Asociada al CSIC, Plaza San Juan 1, 44001 Teruel, Spain

9 University of Michigan, Department of Astronomy, 1085 South University Ave., Ann Arbor, MI 48109, USA

10 University of Alabama, Department of Physics and Astronomy, Gallalee Hall, Tuscaloosa, AL 35401, USA

11 Instituto de Astrofísica de Andalucía, Glorieta de la Astronomía s/n, 18008 Granada, Spain

12 Instituto de Astronomia, Geofísica e Ciências Atmosféricas, Universidade de São Paulo, Rua do Matão 1226, C. Universitária, 05508090 São Paulo, Brazil

13 Departamento de Ciencias Físicas, Universidad Andrés Bello, Fernández Concha 700, Las Condes, Santiago, Chile

14 Departamento de Física, Universidade Federal de Sergipe, Av. Marechal Rondon, s/n. São Cristóvão, 49100-000 Sergipe, SE, Brazil

15 Center for Space Science and Technology, University of Maryland, Baltimore County, 1000 Hilltop Circle, Baltimore, MD 21250, USA

16 European Southern Observatory, Alonso de Cordova 3107, Vitacura, Santiago, Chile

17 Physics Department, Lancaster University, C35, Bailrigg, Lancaster LA1 4YW, UK

\section{Appendix A: Virtual Observatory compliant online catalogue}

In order to help the astronomical community in using our catalogue of candidate UCDs, we developed an archive system that can be accessed from a webpage ${ }^{15}$ or through a Virtual Observatory ConeSearch ${ }^{16}$. The archive system implements a very simple search interface that allows queries by coordinates and radius as well as by other parameters of interest. The user can also select the maximum number of sources (with values from 10 to unlimited) and the number of columns to return (minimum, default, or maximum verbosity). The result of the query is an HTML table with all the sources found in the archive that fulfil the search criteria. The result can also be downloaded as a VOTable or a CSV file. Detailed information on the output fields can be obtained by placing the mouse over the question mark located close to the name of the column. The archive also implements the SAMP ${ }^{17}$ (Simple Application Messaging) Virtual Observatory protocol. SAMP allows Virtual Observatory applications to communicate with each other in a seamless and transparent manner for the user. In this way, the results of a query can be easily transferred to other VO applications, such as Topcat.

\footnotetext{
15 http://svo2.cab.inta-csic.es/vocats/v3/ucd_jplus/ index.php

16 e.g. http://svo2.cab.inta-csic.es/vocats/v3/ucd jplus/cs . php?RA=21.707\&DEC=33.449\&SR=0.1

17 http://www . ivoa. net/documents/SAMP
} 